\title{
Adenoid cystic carcinoma of the larynx: A report of two cases
}

\author{
GUANQIAO LI ${ }^{1}$, JUNNI CHEN $^{2}$, SHUAI ZHANG ${ }^{2},{\text { JIE } \text { LIN }^{2}, \text { FANZHONG KONG }^{2}, \text { FEI CAI }^{3} \text { and SHIPING YANG }}^{2}$ \\ Departments of ${ }^{1}$ Breast Surgery, ${ }^{2}$ Radiation Oncology and ${ }^{3}$ Ear, Nose and Throat, \\ Hainan Province People's Hospital, Haikou, Hainan 570311, P.R. China
}

Received September 16, 2014; Accepted June 11, 2015

DOI: $10.3892 / \mathrm{ol} .2015 .3550$

\begin{abstract}
Laryngeal adenoid cystic carcinoma (ACC) is extremely rare, worldwide. From January 1994 to January 2014, all cases of laryngeal ACC that were diagnosed in the four largest hospitals in Hainan province, were reviewed. Only two such cases were identified. The first patient had a tumor in the subglottic region and the second patient, in the glottic region. The patient with subglottic ACC, who had experienced ongoing symptoms for 3 years, had previously been diagnosed with asthma, at a local hospital. Both presented at an advanced stage. The patient with subglottic disease received a total laryngectomy with a positive surgical margin, was treated with adjuvant radiotherapy, and later succumbed to a pleural effusion as a result of pulmonary metastases. The patient with glottic disease received a partial laryngectomy and declined adjuvant radiotherapy. Subsequently, she developed recurrent disease and passed away following an episode of asphyxia at 14 months post-surgery. Each of these cases had a poor prognosis at presentation. For patients with locoregionally advanced laryngeal ACC, more effective management strategies are required.
\end{abstract}

\section{Introduction}

Malignant tumors of minor salivary glands are uncommon and comprise $2-4 \%$ of all head and neck cancers (1). Minor salivary gland tumors are most common in the oral cavity, in particular in the hard palate, while they occur less commonly in the paranasal sinuses, pharynx, nasal cavity and larynx, as a result of the different distribution of minor salivary glands in these regions (2). Within the larynx, minor salivary gland tumors are rare and represent $<\%$ of laryngeal tumors. They arise from submucosal minor salivary glands, which have a distinct anatomical distribution in the larynx (3).

Laryngeal adenoid cystic carcinomas (ACC) arise from subepithelial glands (4) and 2/3 of these tumors occur in the

Correspondence to: Dr Shiping Yang, Department of Radiation Oncology, Hainan Province People's Hospital, 19 Ziuhua Road, Xiuying District, Haikou, Hainan 570311, P.R. China

E-mail: shipingyang1982@sina.com

Key words: larynx, adenoid cystic carcinoma, poor prognosis subglottis (5). They commonly spread by perineural extension and frequently recur following initial treatment. The etiology of ACC remains unknown. ACC of the larynx is more common in middle-aged and older patients (6). There is no significant difference in the frequency of laryngeal ACC between male and female patients (7).

$\mathrm{ACC}$ of the larynx has been reported in other provinces in China, including Zhejiang (8) and Jiangsu (9), while to the best of our knowledge, there are no reports of this disease from Hainan province to date. In this study, the data of all patients histopathologically diagnosed with laryngeal carcinoma between January 1994 and 2014 at Hainan Province People's Hospital (Haikou, China) were retrospectively reviewed. During this period only two cases of laryngeal ACC were diagnosed, which are discussed in the current report. The anatomical sites of laryngeal ACC were the subglottic and the glottic regions. The two patients had a poor prognosis at presentation. Written informed consent was obtained from the patients' families.

\section{Case report}

Case one (subglottic tumor). A 40-year-old female presented with a 36-month history of increasing progressive dyspnea and cough without hoarseness. She was diagnosed with bronchial asthma at a local hospital. She received antibiotics and anti-asthma treatments (including steroids and bronchodilators) on four occasions, which resulted in some improvement of her symptoms, although they were not fully controlled. Subsequently, she experienced worsening symptoms and became unable to lie supine at night. She presented to the respiratory department of the Hainan Province People's Hospital (Haikou, China), where bronchial asthma was again suspected. She described no relevant medical history and did not report consumption of tobacco or alcohol. Physical examination revealed an increased respiratory rate with diffusing, expiratory wheezing and stridor. No palpable lymph nodes were present in the neck. Pulmonary function tests demonstrated bronchodconstriction (FEV1<80\% of the predicted value). A bronchodilation test was positive. Indirect bronchoscopy revealed a submucosal bulging mass in the posterior wall of the subglottic and upper tracheal region, which measured $3 \mathrm{~cm}$ at its maximum diameter. The lumen of the larynx and upper trachea was markedly obstructed. The mucous membrane surface of the tumor was smooth (Fig. 1A). A subsequent computed tomography (CT) scan of the neck identified a large, subglottic mass that extended to and completely encircled the 

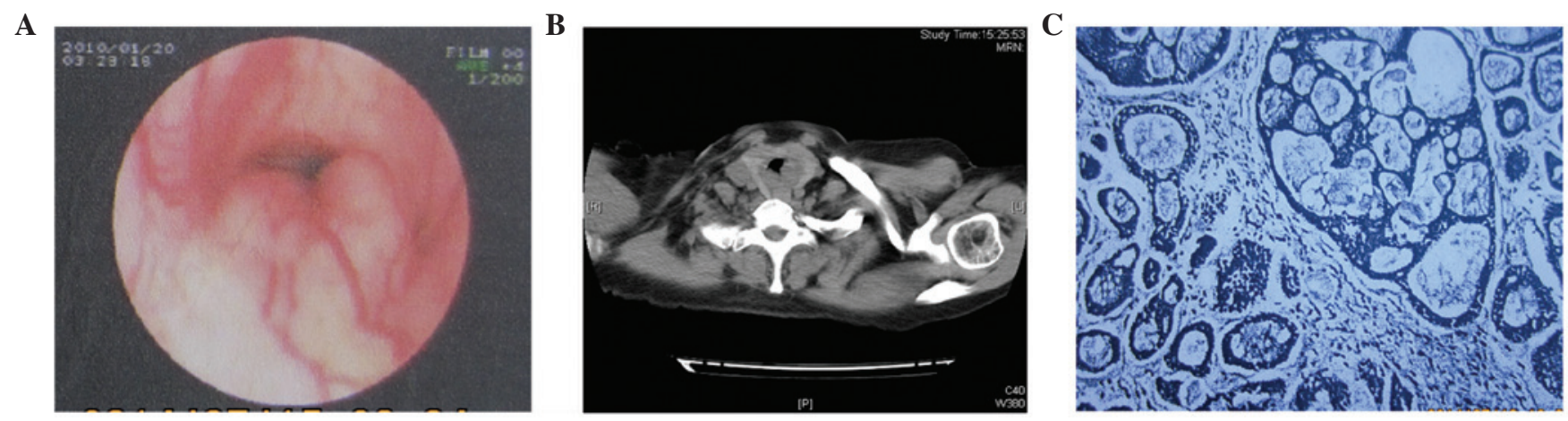

Figure 1. Bronchoscopic, CT and histological examination of case one (subglottic tumor). (A) Bronchoscopic detection of a subglottic mass. (B) CT scan of the larynx, demonstrating a subglotic submucosal mass. (C) Median power view (original magnification, x50) showing a mixed tubular and cribriform pattern of laryngeal adenoid cystic carcinoma (hematoxylin staining). CT, computed tomography.
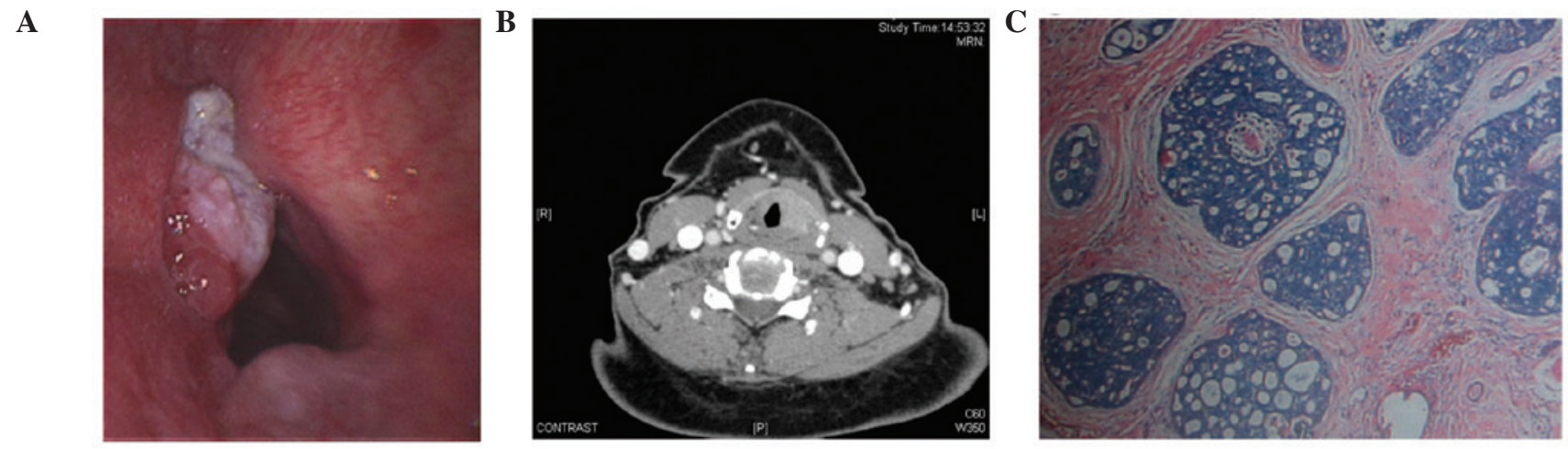

Figure 2. Bronchoscopic, CT and histological examination of case two (glottic tumor). (A) Bronchoscopic detection of a subglottic mass with an uneven surface. (B) CT scan of the larynx, demonstrating a submucosal mass in the left vocal fold. (C) Median power view (original magnification, x50) showing a predominantly cribiform pattern of laryngeal adenoid cystic carcinoma (hematoxylin and eosin staining). CT, computed tomography.

trachea, causing narrowing of the airway (Fig. 1B). No evidence of enlarged neck nodes was detected on CT scanning. Findings on chest $\mathrm{CT}$, bone scan and abdominal ultrasound were normal. During direct microlaryngoscopy, a biopsy was taken from the tumor, and the pathologist reported that the specimen had a mixed tubular and cribriform pattern, which was consistent with the diagnosis of ACC (Fig. 1C). Total laryngectomy, thyroidectomy and partial tracheaectomy without bilateral neck dissections, were performed. Histological examination demonstrated ACC arising in the subglottic region, infiltrating the full thickness of the posterior wall. The anterior, posterior and inferior surgical margins were positive. The superior surgical margin, epiglottis, scutum and thyroid were free from tumor. In view of the histological findings and pre-operative examination, the patient was diagnosed with clinical stage IVa (T4a, N0, M0) disease, according to the Union for International Cancer Control/American Joint Committee on Cancer staging system (10). Following surgery, the patient received adjuvant radiation therapy. The patient was treated with 3 -dimensional conformal radiotherapy (3-DCRT). The accumulated dose was $71.3 \mathrm{~Gy}(2.3 \mathrm{~Gy} /$ fraction $\mathrm{x} 31$ fractions) to the affected areas. The patient was followed up with clinic visits and physical examinations every 3 months. She developed multiple lung and bone metastasis without locoregional recurrence at 15 months post-surgery. The patient succumbed to a malignant pleural effusion as a result of pulmonary metastasis at 34 months post-surgery.
Case two (glottic tumor). A 44-year-old woman with a 12-month history of progressive hoarseness and a 4-month history of progressive dyspnea was referred to the ear, nose and throat department of Hainan Province People's Hospital.

Fiberoptic laryngoscopy identified a swelling of the left vocal cord with an uneven surface, which extended forwards to the anterior commissure (Fig. 2A). A CT scan revealed a mass involving the left vocal cord and anterior commissure, and infiltrating the left paraglottic space (Fig. 2B). Neither physical examination nor neck CT detected any tumescent lymph nodes in the neck, and nothing abnormal was detected on chest X-ray, bone scan or abdominal ultrasound. According to the clinical and radiological data, total laryngectomy was proposed. The patient declined this intervention and therefore underwent a partial laryngectomy without neck dissection. The final pathological report showed negative margins. The patient was diagnosed with stage III (T3, N0, M0) disease, according to the histological findings and pre-operative examination (10). Following surgery, the patient declined adjuvant radiation therapy. An MRI scan at 6 months demonstrated relapse of the tumor. The patient declined an expanded total laryngectomy and succumbed to asphyxia at 14 months post-surgery.

\section{Discussion}

A review of the literature identified 175 well-documented cases of ACC of the larynx. Morais Pérez et al (11) reported 80 cases 
up to 1999 and Del Negro et al (12) reported 120 cases up to 2007. After 2007, only a further 55 cases have been reported in the literature.

Laryngeal carcinoma is divided into 3 subtypes, supraglottic, glottic and subglottic, according to origin. The signs and symptoms of laryngeal ACC are correlated with tumor location and size. Tumors of the supraglottic region present with dysphagia. Hoarseness or dyspnea, is indicative of glottic involvement. The majority of patients with supraglottic or glottic tumors are diagnosed at an early stage as symptoms are identified early (2). However, Hainan province is less advanced in terms of medical technology, compared with other provinces in China. Thus, numerous patients in Hainan province do not visit a doctor until they have developed obvious intrusive symptoms. Thus, as observed in this study, the patient with the glottic tumor (case two) presented at an advanced stage of disease.

Stridor and airway obstruction are more frequently associated with subglottic tumors. As laryngeal ACC spreads in a submucosal fashion, it is difficult to detect at an early stage of disease. This explains why the majority of patients are diagnosed at an advanced stage $(2,13,14)$. For these reasons, the high number of late diagnoses may be fatal in some cases (12). In this study, the patient with advanced stage subglottic disease (case one), presented with symptoms of dyspnea, cough and stridor, which were diagnosed as asthma, on multiple occasions over a period of 3 years in a local hospital. Misdiagnosis is common as dyspnea, cough and stridor are also symptoms of asthma.

The histopathological pattern of ACC is classified into three distinct subtypes: Cribriform, which is the most common form; tubular, which has the best prognosis; and solid, which carries the worst prognosis (12). In the present case, microscopic examination demonstrated a classic cribriform pattern in the tumor in case two and a mixed tubular and cribriform pattern in case one, neither of which are considered to predict the worst prognosis patients with laryngeal ACC.

Surgical excision is recommended due to the relative radioresistance of these tumors (2). As a result of the propensity for submucosal spread, in addition to perineural and lymphovascular invasion, total laryngectomy is often required (2).

Postoperative radiation is advocated by Coca-Pelaz et al (15), since radiotherapy has been shown to result in tumor regression and symptomatic relief (15-17). The use of chemotherapy in ACC remains controversial. A number of studies have reported positive responses to chemotherapy and it is recommended as palliative therapy in cases of advanced disease (16). The role of chemotherapy in ACC of the head and neck is usually confined to advanced (nonresectable), recurrent, or metastasic disease. From a review of the efficacy of various chemotherapy regimens, either as monotherapy or in combination schedules, Papaspyrou et al concluded that response rates to chemotherapy are low and response duration is generally short lived. Based on the available data, no standard chemotherapy regimen could be recommended (18).

In view of the extreme rarity of laryngeal ACC, it is difficult to conduct clinical trials. The optimum treatment regimes remain unclear. Previous case reports recommend different management strategies and there are few data on the long-term results of these approaches $(8,12,13,19)$. There is not yet a standardized treatment for the management of patients with laryngeal ACC.
In the patient with subglottic disease, presented here, the surgical margin was positive. This patient therefore was at high risk of a relapse. However, she showed no local recurrence prior to death. This may have been due to treatment with high-dose radiotherapy. Whilst this patient did not have evidence of local recurrence, she succumbed to a pleural effusion resulting from pulmonary metastases at 34 months post-surgery. Therefore, chemotherapy may be useful as adjuvant therapy in high-risk patient, in order to prevent distant metastasis.

In the case of laryngeal ACC involving the glottic region, the tumor infiltrated the paraglottic space and the patient underwent a partial laryngectomy. The patient declined adjuvant radiation therapy. She developed relapse of the disease at 6 months post-surgery.

Squamous cell carcinoma is the commonest pathological type of laryngeal carcinoma. Five-year overall survival rates for patients with advanced laryngeal squamous cell carcinoma range from 48-54\% (20-22). The five-year survival rates for patients with laryngeal ACC have been reported to range from $12-17 \%$, following surgery $(12,13)$. Therefore, more effective management strategies for laryngeal ACC are required.

In conclusion, laryngeal ACC is rare in Hainan province and worldwide. In the two cases presented here, the diagnosis was made at an advanced stage. Each of these cases had a poor prognosis at presentation. It its likely that early diagnosis may improve survival. Furthermore, wide-margin surgery in combination with post-operative radiotherapy for advanced lesions with perineural spread, or close or positive margins, is essential. Chemotherapy may be useful as adjuvant therapy in high-risk patients in order to prevent distant metastasis.

\section{References}

1. Sadeghi A, Tran LM, Mark R, Sidrys J and Parker RG: Minor salivary gland tumors of the head and neck: Treatment strategies and prognosis. Am J Clin Oncol 16: 3-8, 1993.

2. Ganly I, Patel SG, Coleman M, Ghossein R, Carlson D and Shah JP: Malignant minor salivary gland tumors of the larynx. Arch Otolaryngol Head Neck Surg 132: 767-770, 2006.

3. Bak-Pedersen K and Nielsen KO: Subepithelial mucous glands in the adult human larynx. Studies on number, distribution and density. Acta Otolaryngol 102: 341-352, 1986

4. Tincani AJ, Del Negro A, Araújo PP, et al: Management of salivary gland adenoid cystic carcinoma: Institutional experience of a case series. Sao Paulo Med J 124: 26-30, 2006.

5. Dexemble P, Huth J, Rebufy M and Chabrol A: Cystic adenoid carcinoma of the larynx: Two cases. Ann Otolaryngol Chir Cervicofac 120: 244-248, 2003 (In French).

6. Tewfik TL, Novick WH and Schipper HM: Adenoid cystic carcinoma of the larynx. J Otolaryngol 12: 151-154, 1983.

7. Lee LA, Fang TJ, Li HY and Lee KF: Adenoid cystic carcinoma of the supraglottis mimicking a laryngeal cyst. Otolaryngol Head Neck Surg 129: 157-158, 2003.

8. Wang HL, Xu L and Li FJ: Subglottic adenoid cystic carcinoma mistaken for asthma. J Zhejiang Univ Sci B 10: 707-710, 2009.

9. Qian X, Zhou H, Gu Y, Zhang Y and Gao X: Supraglottic adenoid cystic carcinoma mimicking laryngeal amyloidosis: A case report. Oncol Lett 7: 2154-2156, 2014.

10. Edge SB, Byrd DR, Compton CC, Fritz AG, Greene FL and Trotti A III: editors. Larynx AJCC Cancer Staging Manual. 7th ed. New York: Springer pp57-62, 2010.

11. Morais Pérez D, Cortejoso Hernández A, Borau Clavero M and Martín Sigüenza G: Carcinoma adenoide quístico de laringe. Adenoid cystic carcinoma of the larynx. Acta Otorrinolaringol Esp 50: 660-663, 1999 (In Spanish).

12. Del Negro A, Ichihara E, Tincani AJ, Altemani A and Martins AS: Laryngeal adenoid cystic carcinoma: Case report. Sao Paulo Med J 125: 295-296, 2007. 
13. Zvrko E and Golubović M: Laryngeal adenoid cystic carcinoma. Acta Otorhinolaryngol Ital 29: 279-282, 2009.

14. Testa D, Guerra G, Conzo G, Nunziata M, D'Errico G, Siano M, Ilardi G, Vitale M, Riccitiello F and Motta G: Glottic-Subglottic adenoid cystic carcinoma. A case report and review of the literature. BMC Surg 13 (Suppl 2): S48, 2013.

15. Coca-Pelaz A, Rodrigo JP, Bradley PJ, Vander Poorten V, Triantafyllou A, Hunt JL, Strojan P, Rinaldo A, Haigentz M Jr, Takes RP, et al: Adenoid cystic carcinoma of the head and neck - An update. Oral Oncol 51: 652-661, 2015.

16. Kokemueller H, Eckardt A, Brachvogel P and Hausamen JE: Adenoid cystic carcinoma of the head and neck - a 20 years experience. Int J Oral Maxillofac Surg 33: 25-31, 2004

17. Subramaniam T, Lennon P and O'Neill JP. Ongoing challenges in the treatment of adenoid cystic carcinoma of the head and neck. Ir J Med Sci: Apr 17, 2015 (Epub ahead of print).

18. Papaspyrou G, Hoch S, Rinaldo A, et al: Chemotherapy and targeted therapy in adenoid cystic carcinoma of the head and neck: a review. Head Neck 33: 905-911, 2011.
19. Saraydaroglu O, Coskun $\mathrm{H}$ and Kasap M: Unique presentation of adenoid cystic carcinoma in postcricoid region: A case report and review of the literature. Head Neck Pathol 5: 413-415, 2011.

20. Mantsopoulos K, Psychogios G, Bohr C, Zenk J, Kapsreiter M, Waldfahrer $\mathrm{F}$ and Iro H: Primary surgical treatment of T3 glottic carcinoma: Long-term results and decision-making aspects. Laryngoscope 122: 2723-2727, 2012.

21. Ganly I, Patel SG, Matsuo J, Singh B, Kraus DH, Boyle J, Wong RJ, Lee N, Pfister DG, Shaha AR, et al: Predictors of outcome for advanced-stage supraglottic laryngeal cancer. Head Neck 31: 1489-1495, 2009.

22. Nguyen-Tan PF, Le QT, Quivey JM, Singer M, Terris DJ, Goffinet DR and Fu KK: Treatment results and prognostic factors of advanced T3-4 laryngeal carcinoma: The University of California, San Francisco (UCSF) and Stanford University Hospital (SUH) experience. Int J Radiat Oncol Biol Phys 50: 1172-1180, 2001. 